\title{
The nasal tent: an adjuvant for performing endoscopic endonasal surgery in the Covid era and beyond
}

\author{
S. H. Maharaj ${ }^{1}$ (D) \\ Received: 6 June 2020 / Accepted: 16 June 2020 / Published online: 23 June 2020 \\ c) Springer-Verlag GmbH Germany, part of Springer Nature 2020
}

\begin{abstract}
Purpose To propose a cost-effective reproducible barrier method to safely perform endoscopic endonasal surgery during the Covid-19 pandemic.

Methods This manuscript highlights the use of a clear, cost-effective disposable plastic sheet that is draped as a tent over the operating area to contain aerolization of particles. This is then connected to a suction to remove airborne particles and thus reduce transmission of the virus.

Conclusion The use of a nasal tent is a simple and affordable method to limit particle spread during high-risk aerolisation procedures during the Covid era and beyond.
\end{abstract}

Keywords Nasal tent; endoscopic sinus surgery · Covid-19

\section{Background}

The advent of the Covid-19 pandemic has disrupted healthcare systems and surgical procedures throughout the world. Coronaviruses are approximately $0.125 \mu \mathrm{m}$ in size and are frequently carried in respiratory droplets [1]. The study demonstrates that COVID-19 can remain viable and infectious for hours in aerosolized materials and for days on surfaces [2].

Due to the high risk of viral aerolisation during procedures such as endoscopic sinus surgery, a plethora of cases have been postponed or cancelled [3].

However, it is not feasible to continue in this manner, as the duration of the pandemic is hard to predict. There may also be further global viral pandemics in future and thus the otorhinolaryngology community has to now adapt to the new normal. Mitigation strategies have included cold surgical instrumentation, negative pressure theatres and the use of the microdebrider [4].

The gold standard would be to create two self-contained surgical environments (one for the aneathetized patient and

\section{S. H. Maharaj}

Shivesh.maharaj@wits.ac.za

1 Department of Otorhinolaryngology, University of the Witwatersrand, Johannesburg, South Africa another for the theatre team) that can easily interact with each other and not allow cross contamination.

The use of Personal protective equipment (PPE) creates a barrier that protects the health care worker, however, it would be easier just to isolate the patient within a threedimensional surgical field that would trap and remove aerosolized particles [5].

We propose the use of a simple clear isolation plastic sheet $(160 \times 200 \mathrm{~cm})$ nasal tent to limit aerolization during these procedures. The cost of such a sheet varies from 4 to 8 US dollars and is quite readily available in most centres throughout the world.

\section{Description of the technique}

The patient is intubated with a cuffed endotracheal tube using PPE and the video laryngoscope. The patient is positioned, a throat pack inserted and draped. An operating tray is positioned at the cranial end of the bed $30 \mathrm{~cm}$ above the patients head (Fig. 1).

Two openings are left for the surgeons hands and the scrub nurse. These are inserted under the tent which then secured to the operating table using adhesive tape along with the entire head and torso region. A separate low flow suction unit (such that it does not collapse the tent) is connected to the tent to steadily remove aerosolized particles within the tent and filter 


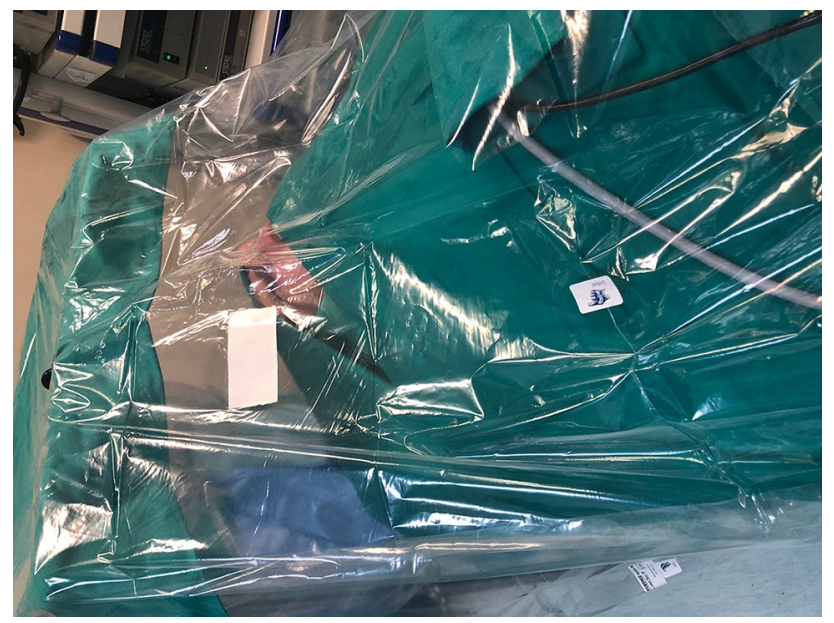

Fig. 1 Nasal tent draped over the patient

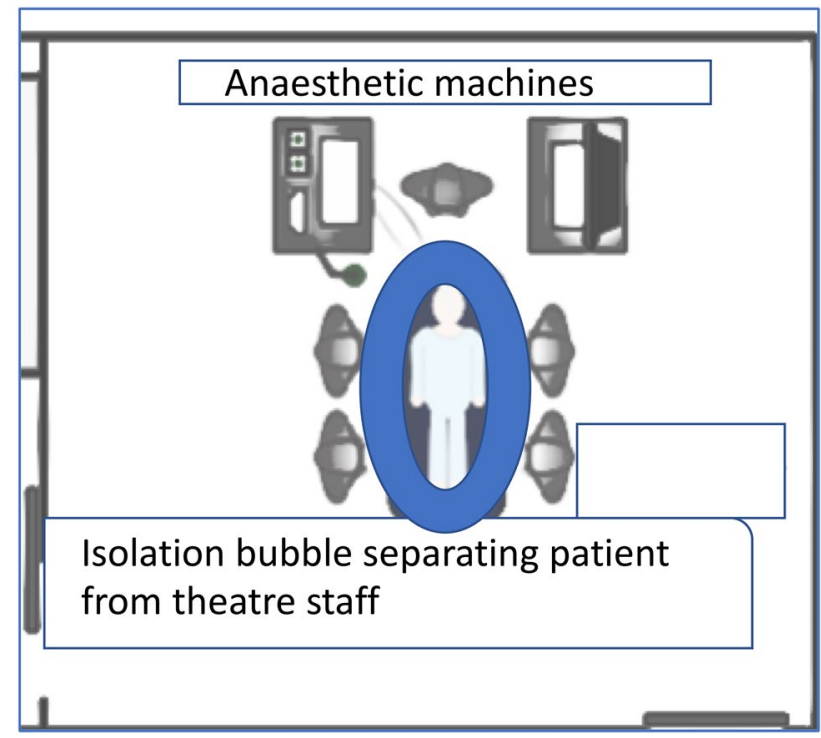

Fig. 2 Diagram of the isolated patient in theatre-bubble theory

it through a separate suction unit to prevent cross-contamination with the theatre filtration system (Fig. 2).

\section{Indications}

Endoscopic sinonasal procedures done in the operating theatre.

\section{Limitations}

The tent is best suited for a single surgeon procedure. Once the procedure is started there is no passing of instruments under the tent, thus all instruments need to be selected and set out under the tent prior to surgery.
There is a learning curve to operating under the tent.

The theatre staff still need to use full PPE.

Currently may only be used in theatre.

Further studies are required to assess the efficacy and efficiency of the nasal tent.

How to avoid complications:

Theatre staff should be trained and should practice on how to safely drape and remove the plastic without compromising the integrity of the unit.

The use of diathermy and sharp instrumentation needs to be carefully monitored so as to not damage the nasal tent and compromise its barrier effect.

The surgery should be well planned with all members of the team.

All surgical instruments and apparatus should be secured into the tent prior to commencing the procedure. Once the surgeon has placed his hands under the tent, the adhesive tape creates a seal that prevents aerosolization into the operating theatre.

\section{Key points}

- Pre-operative testing of surgical patients prior to endonasal surgery is advised.

- Postpone surgery in COVID-19 positive patients if possible till they recovered.

- All emergency cases and untested patients should be managed as positive until proven otherwise.

- Aneasthesia and surgery should limit the degree of aerolization of particles.

- Cost-Effective and simple barriers may be used to isolate the surgical field and prevent contamination of the theatre and infection of theatre staff.

- The nasal tent is an easy to learn and cost-effective barrier that may be used to prevent viral dissemination in theatre.

- Further by connecting suction to the tent will the viral particles may be safely extracted and filtered.

- The Covid pandemic may be protracted and thus we will have to re-think the way we perform surgery to protect both the patient and the staff, in the current pandemic climate and beyond.

Funding No research grant was utilized for the study conducted.

\section{Compliance with ethical standards}

Conflict of interest The authors hereby declare that they have no conflict of interest. 
Ethical approval All procedures performed in this study involving human participants were conducted in accordance with the ethical standards of the Helsinki guidelines. Consent was obtained from the patient for the use of the images.

\section{References}

1. Fehr AR, Perlman S (2015) Coronaviruses: an overview of their replication and pathogenesis. Methods Mol Biol 1282:1-23

2. Van Doremalen N, Bushmaker T, Morris DH, Holbrook MG, Gamble A, Williamson BN et al (2020) Aerosol and surface stability of SARS-CoV-2 as compared with SARS-CoV-1. N Engl J Med. 382:1564-1567

3. Judson SD, Munster VJ (2019) Nosocomial transmission of emerging viruses via aerosol-generating medical procedures. Viruses 11:940
4. Zheng MH, Boni L, Fingerhut A (2020) Minimally invasive surgery and the novel coronavirus outbreak: lessons learned in China and Italy. Ann Surg 272(1):e5-e6. https://doi.org/10.1097/ SLA.0000000000003924

5. Radonovich LJ Jr, Simberkoff MS, Bessesen MT, Brown AC, Cummings DAT, Gaydos CA et al (2019) N95 respirators vs medical masks for preventing influenza among health care personnel: a randomized clinical trial. JAMA 322(9):824-833

Publisher's Note Springer Nature remains neutral with regard to jurisdictional claims in published maps and institutional affiliations. 